Research Paper

\title{
miR-135b-5p inhibits LPS-induced TNF $\alpha$ production via silencing AMPK phosphatase Ppm1e
}

\author{
Ping Li ${ }^{1}$, Jian-bo Fan ${ }^{2}$, Yanxia Gao ${ }^{1}$, Ming Zhang ${ }^{1}$, Li Zhang ${ }^{1}$, Ning Yang ${ }^{1}$, Xiaojing \\ Zhao $^{1}$ \\ ${ }^{1}$ Department of Emergency, the Second Affiliated Hospital of Xi'an Jiao Tong University, Xi'an, China \\ ${ }^{2}$ Department of Orthopaedics, The Second Affiliated Hospital of Nantong University, Nantong, China \\ Correspondence to: Xiaojing Zhao, email: Zhao_xiandr@163.com
}

Keywords: miR-135b-5p, Ppmle, AMPK, LPS, TNF $\alpha$

Received: September 02, 2016

Accepted: October 05, 2016

Published: October 25, 2016

\section{ABSTRACT}

\begin{abstract}
AMPK activation in monocytes could suppress lipopolysaccharide (LPS)induced tissue-damaging TNF $\alpha$ production. We are set to provoke AMPK activation via microRNA ("miRNA") downregulating its phosphatase Ppm1e. In human U937 and THP-1 monocytes, forced expression of microRNA-135b-5p ("miR-135b-5p") downregulated Ppm1e and activated AMPK signaling. Further, LPS-induced TNFa production in above cells was dramatically attenuated. Ppm1e shRNA knockdown in U937 cells also activated AMPK and inhibited TNFa production by LPS. AMPK activation is required for miR-135b-induced actions in monocytes, AMPKa shRNA knockdown or T172A dominant negative mutation almost abolished miR-135b-5p's suppression on LPS-induced TNFa production. Significantly, miR-135b-5p inhibited LPS-induced reactive oxygen species (ROS) production, NFKB activation and TNFa mRNA expression in human macrophages. AMPKa knockdown or mutation again abolished above actions by $\mathrm{miR}-135 \mathrm{~b}-5 \mathrm{p}$. We conclude that $\mathrm{miR}-135 b-5 p$ expression downregulates Ppm1e to activate AMPK signaling, which inhibits LPS-induced TNFa production via suppressing ROS production and NFKB activation.
\end{abstract}

\section{INTRODUCTION}

Chronic obstructive pulmonary disease (COPD) patients suffer chronic and consistent airway inflammations [1-3]. A significant increased level of circulating pathogen-associated molecular patterns (PAMPs) is detected in COPD patients' lungs [1-3]. Lipopolysaccharide (LPS), one of the most prominent PAMPs, activates resident monocytes and induces production pro-inflammatory cytokines (i.e. tumor necrosis factor- $\alpha$ or TNF $\alpha$ ) [4, 5]. TNF $\alpha$ level is significantly elevated in COPD patients' bronchoalveolar lavage fluids, sputum, as well as plasma and lung tissues [6-8]. Anti$\mathrm{TNF} \alpha$ strategy was applied to attenuate COPD patients' inflammations [6-8]. Our group has been focusing on the underlying mechanisms of LPS-induced TNF $\alpha$ production in monocytes [9], which might help to develop possible intervention measures [9].

AMP-activate protein kinase (AMPK) plays a pivotal role in maintaining cellular energy balance [10].
Recent studies have discovered the important function of this kinase in regulating inflammatory responses [11-14]. For instance, two well-known AMPK activators, AICAR and A769662, were shown to inhibit LPS-induced nuclear factor kappa $\mathrm{B}(\mathrm{NF} \mathrm{B})$ activation and pro-inflammatory cytokine production $[11,12]$. Ducommun et al., showed that metformin activated AMPK signaling to inhibit cytokine-induced pro-inflammatory responses [15]. Shen et al., showed that perifosine surprisingly reduced LPSinduced TNF $\alpha$ production via activating AMPK [12]. Our unpublished results recently found that GSK621, a novel AMPK activator [16], inhibited LPS-induced $\mathrm{TNF} \alpha$ production in macrophages/monocytes (Wu et al., unpublished results). Therefore, targeted-activation of AMPK could be a novel strategy to inhibit LPS-induced inflammatory responses $[11,12,15,17]$.

Thr172 phosphorylation of AMPK $\alpha$ subunit is vital for AMPK activation [10, 18, 19]. Numerous studies have focused on the mechanisms of kinase phosphorylation of this site [20]. Several AMPK kinases (i.e. LKB1 [19], 
CaMKK [21] and TAK1 [22]) have been characterized thus far. Yet, the phosphatase of AMPK $\alpha$-Thr172 is largely unknown. A recent study by Voss et al., has proposed that Ppmle could be a key AMPK $\alpha$ phosphatase [23]. Ppm1e depletion, inhibition or mutation was able to induce AMPK $\alpha$-Thr172 phosphorylation and AMPK activation [23].

microRNAs (miRNAs) are capable of decreasing expression of target mRNAs at both post-transcriptional and transcriptional levels [24]. Here we set to indentify miRNA that activates AMPK though specifically targeting Ppm1e. Multiple miRNA databases were searched. We found that microRNA-135b-5p ("miR-135b$5 \mathrm{p}$ ") selectively targets Ppm1e's untranslated regions (UTRs, 3'). Further, forced-expression of miR-135b-5p downregulates Ppm1e to activate AMPK signaling, which subsequently inhibits LPS-induced TNF $\alpha$ production in human monocytes.

\section{RESULTS}

\section{Expression of miR-135b-5p downregulates Ppm1e in human macrophages}

First, miR-135b-5p indeed complements UTRs (position 517-524) of Ppm1e (Figure 1A, which is also seen in the recent study [25]). As described, an expression vector ("pSuper-neo") integrating miR-135b-5p [25] was transfected to U937 macrophages. Via neomycin selection, two stably U937 lines ("Line-1" and "Line-2") with miR-135b-5p were established. Real-time qPCR ("RTqPCR") assay analyzing miR-135b-5p level confirmed the phenotype of two stably cell lines, with high level of miR-135b-5p expression (Figure 1B). Intriguingly, mRNA and protein expressions of Ppm1e were sharply downregulated following miR-135b-5p expression (Figure 1C). The experiments were also repeated in another human macrophage cell line: THP-1. Two stably THP-1 cell lines ("Line-1" and "Line-2") expressing miR-135b-5p (Figure 1D) were established. These cell lines again showed downregulated Ppmle (Figure 1E). Notably, the nonsense control mi-RNA ("miR-C") showed no effect on expression of miR-135b-5p (Figure 1B and 1D) or Ppm1e (Figure $1 \mathrm{C}$ and $1 \mathrm{E}$ ). Together, these results demonstrate that miR-135b-5p targets and downregulates Ppm1e in human macrophages.

\section{miR-135b-5p activates AMPK and inhibits LPS-induced TNF $\alpha$ production in human macrophages}

Previous studies have demonstrated that Ppm1e is an AMPK phosphatase [23]. Above results showed that miR-135b-5p downregulated this phosphatase in human macrophages. Thus AMPK signaling was then tested in these cells. As shown in Figure 2A, the level of phosphorylated ("p") AMPK $\alpha$ (Thr-172) and its major downstream acetyl-CoA carboxylase (ACC, Ser-79) was significantly increased in miR-135b-5p-expressing U937 cells, indicating profound AMPK activation. As discussed, studies have implied that AMPK activation can inhibit LPS-induced inflammatory responses [11-14]. Therefore, the potential effect of miR-135b-5p on LPS-induced TNF $\alpha$ protein was then tested. ELISA assay results in Figure $2 \mathrm{~B}$ showed that LPS-induced TNF $\alpha$ protein secretion was largely inhibited in miR-135b-5p-expressing U937 cells. miR-135b-5p resulted in over $60 \%$ reduction of $\mathrm{TNF} \alpha$ production (Figure 2B). Similar results were also obtained in THP-1 macrophages, where miR-135b-5p induced significant AMPK activation and inhibited LPS-induced $\mathrm{TNF} \alpha$ production (Data not shown).

\section{Ppm1e shRNA knockdown activates AMPK and inhibits LPS-induced TNF $\alpha$ production}

Based on the results above, Ppmle knockdown should also activate AMPK and inhibit TNF $\alpha$ production. Thus, lentiviral shRNA strategy was applied to knockdown Ppm1e in U937 cells. Two stably U937 cell lines with Ppm1e-shRNA ("-1/-2") were established. Western blot results in Figure $3 \mathrm{~A}$ confirmed that Ppmle expression was downregulated in the stably cells. Consequently, AMPK activation (p-AMPK $\alpha$ ) was increased (Figure 3A). Notably, Ppm1e shRNA didn't change miR-135b-5p expression (Figure 3B). Significantly, LPS-induced TNF $\alpha$ production in U937 cells was dramatically attenuated with Ppmle shRNA knockdown (Figure 3C). The scramble non-sense control shRNA ("sh-C") showed no effect on Ppm1e expression, AMPK activation nor TNF $\alpha$ production (Figure 3A and 3B). We repeated the above experiments in THP-1 cells, and similar results were achieved (Data not shown).

If, as we proposed, Ppmle is the primary target of miR-135b-5p in mediating its actions in monocytes, miR135b-5p should possibly be invalid in Ppmle-depleted cells. We thus expressed miR-135b-5p in Ppm1e-shRNA expressing U937 cells. RT-qPCR assay results confirmed miR-135b-5p over-expression (Figure 3D) in the Ppm1esilence U937 cells (Figure 3E). Importantly, miR-135b$5 \mathrm{p}$ expression failed to further inhibit LPS-induced TNF $\alpha$ production in the Ppm1e-silenced cells ( $p>0.05$, Figure 3F). These results indicate that Ppmle is likely the primary target of miR-135b-5p in mediating its actions against LPS.

\section{AMPK activation is required for miR-135b-5p's inhibition on LPS-induced TNF $\alpha$ production}

If AMPK activation is the primary reason of miR$135 \mathrm{~b}-5 \mathrm{p}$-induced action against TNF $\alpha$ production by 

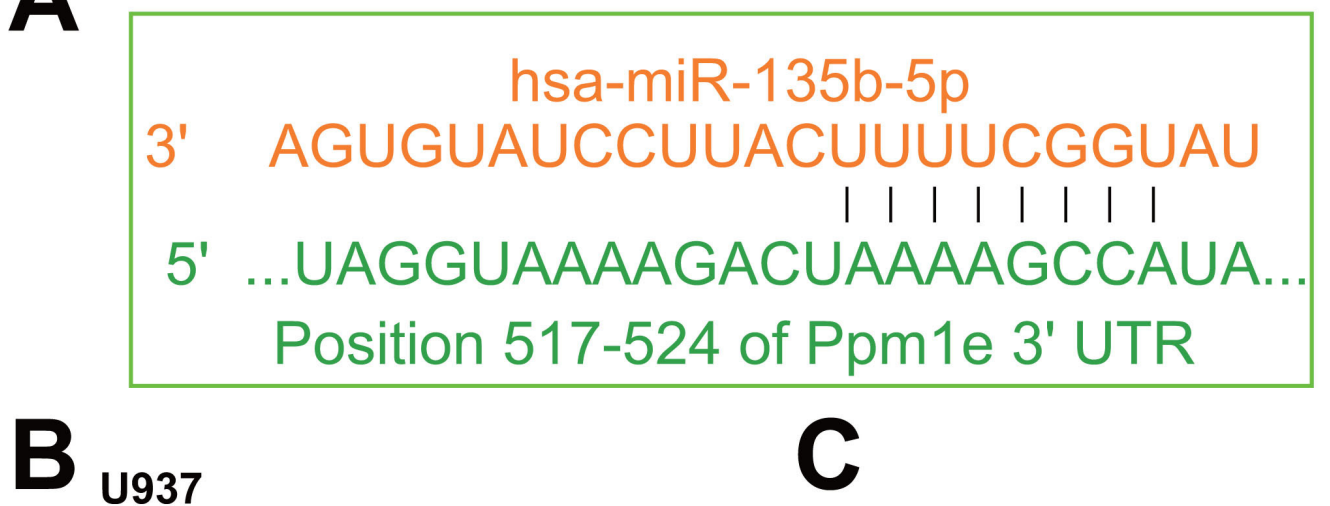

U937
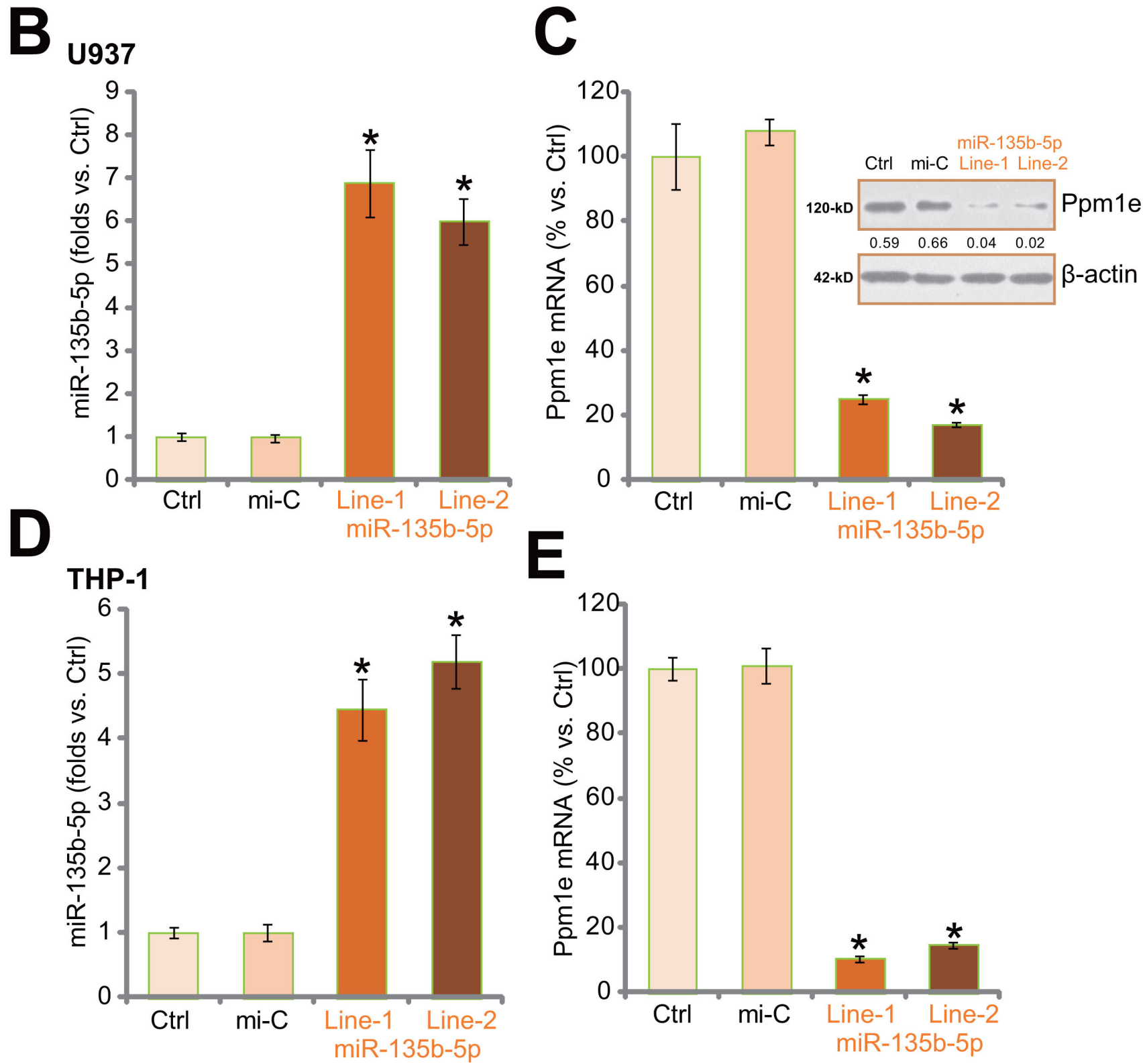

Figure 1: Expression of miR-135b-5p downregulates Ppm1e in human macrophages. A. miR-135b-5p complements Ppm1e's 3' untranslated regions (UTRs, position 517-524) (A). Human U937 or THP-1 macrophages were transfected with miR-135b-5p construct or non-sense control microRNA ("miR-C"), and stable cells were established via neomycin selection. Expression of miR-135b5p B and D. and Ppm1e mRNA (C and E., left panels) were tested by quantitative real-time PCR ("RT-qPCR") assay; Ppm1e protein expression was examined by Western blot assay (C, right panels). Experiments in this figure were repeated three times, and similar results were obtained. Ppm1e protein expression (vs. $\beta$-actin) was quantified (C). "Ctrl" stands for non-transfected control cells (B-E). * $p<0.05$ vs. "miR-C" group (B-E). 


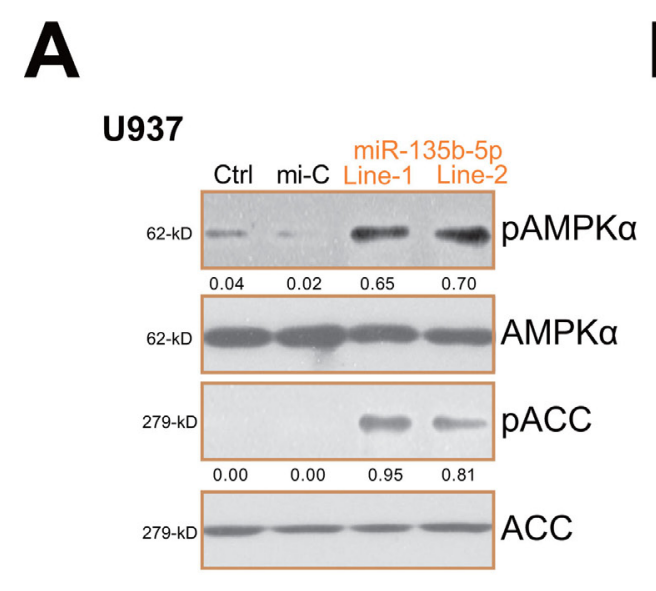

B

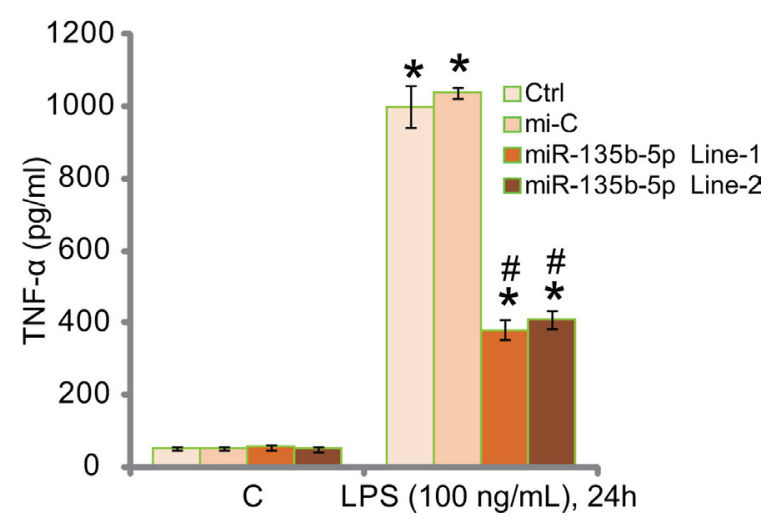

Figure 2: miR-135b-5p activates AMPK signaling and inhibits LPS-induced TNF $\alpha$ production in human macrophages. Stably U937 macrophages expressing miR-135b-5p (two lines, "Line-1/-2") or non-sense control microRNA ("miR-C") were subjected to Western blot assay of phosphorylated- ("p") and regular AMPK $\alpha$ and ACC A. Above cells were treated with LPS (100 ng/mL) or medium control ("C") for 24 hours, TNF $\alpha$ content in conditional medium was tested by ELISA assay B. Experiments in this figure were repeated for three times, and similar results were obtained. AMPK $\alpha$ and ACC phosphorylations were quantified (A). "Ctrl" stands for un-transfected control cells. * $p<0.05$ vs. group "C" (B). " $p<0.05$ vs. LPS treatment of "miR-C" group (B).
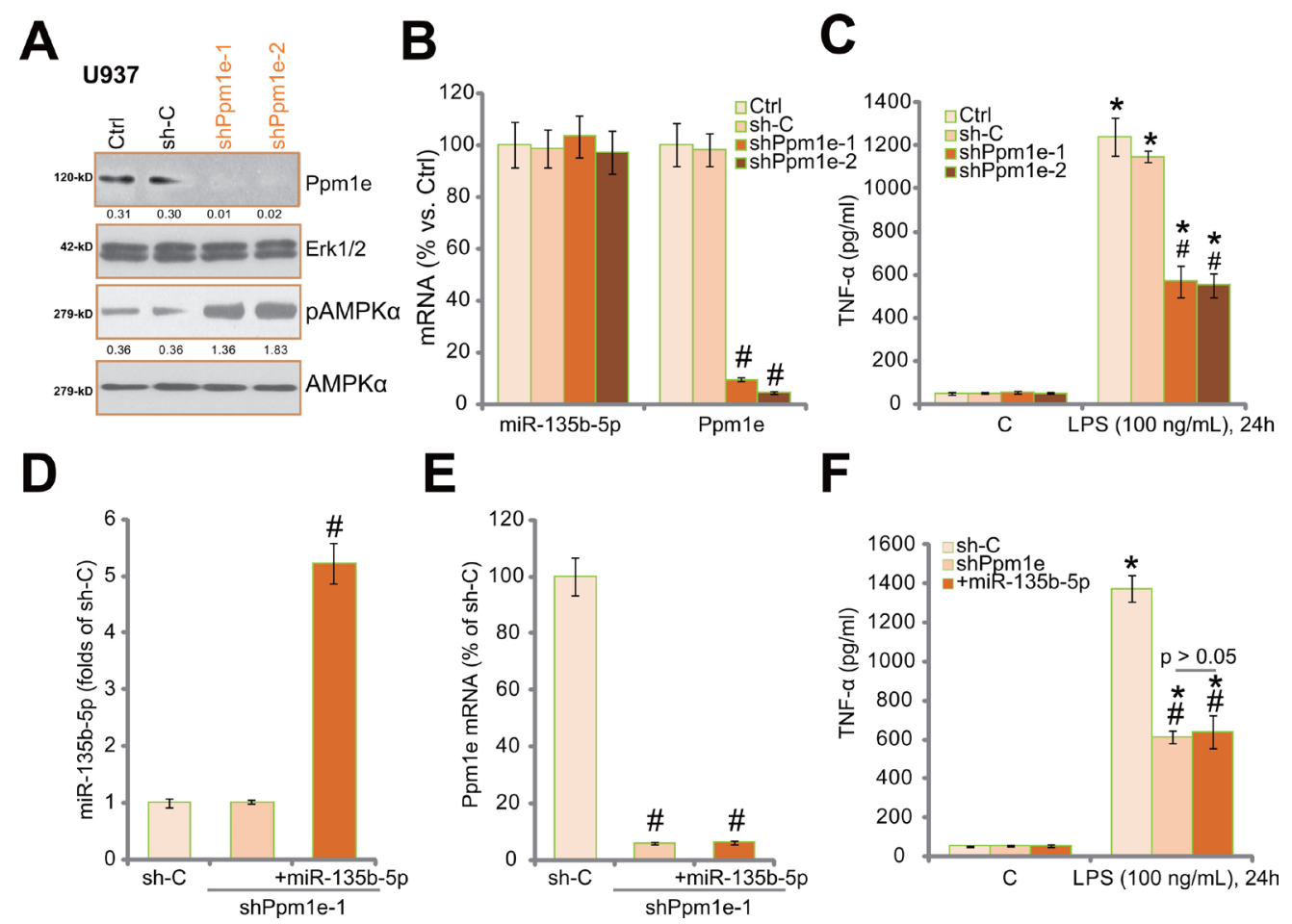

Figure 3: Ppm1e shRNA knockdown activates AMPK and inhibits LPS-induced TNFa production in human macrophages. Stably U937 cells expressing Ppm1e shRNA ("shPpm1e-1" or "shPpm1e-2", with non-overlapping sequences) or scramble control shRNA ("sh-C") were subjected to Western blot assay of listed proteins A. or RT-qPCR assay of miR-135b-5p and Ppmle mRNA B. Above cells were treated with LPS (100 ng/mL) or medium control ("C") for 24 hours, TNF $\alpha$ production was tested by ELISA assay C. U937 cells with shPpmle-1 were also transfected with miR-135b-5p construct, and stably cells were again established; miR-135b$5 p$ D. and Ppmle mRNA E. expressions were tested by RT-qPCR assay. Above cells were treated with LPS $(100 \mathrm{ng} / \mathrm{mL})$ for 24 hours, TNF $\alpha$ production was measured F. Ppmle expression (vs. Erk1/2) and AMPK $\alpha$ phosphorylation were quantified (A). "Ctrl" stands for un-transfected control cells. Experiments in this figure were repeated for three times, and similar results were obtained. " $p<0.05 v$ s. "sh-C" group (B-F). * $p<0.05$ vs. "C" group (C and F). 
LPS, AMPK inhibition should then abolish miR-135b's activity. Genetic strategies were applied. Two different non-overlapping lentiviral AMPK $\alpha$ shRNAs ("No.1" and "No.2") were utilized to knockdown AMPK $\alpha$ in miR-135b-5p-expressing U937 cells (Figure 4A). As a result, miR-135b-5p-induced AMPK activation, or AMPK $\alpha / A C C$ phosphorylation, was dramatically inhibited (Figure 4A). Remarkably, AMPK $\alpha$ shRNAs almost abolished miR-135b-5p-induced inhibition of $\mathrm{TNF} \alpha$ production (Figure 4B). In another words, miR$135 \mathrm{~b}-5 \mathrm{p}$ was in-effective against $\mathrm{TNF} \alpha$ production in AMPK $\alpha$-depleted cells (Figure 4B). Above results suggest that AMPK activation is required for miR-135b$5 \mathrm{p}$-induced actions in monocytes. Net, we introduced a dominant-negative mutant of AMPK $\alpha$ (T172A) into miR-135b-5p-expressing U937 cells [26]. The mutant AMPK $\alpha$ dramatically attenuated AMPK activation (Figure 4C). Significantly, ELISA results in Figure 4D showed that AMPK $\alpha$ mutation almost nullified miR$135 \mathrm{~b}$ 's inhibition on TNF $\alpha$ production by LPS. These two sets of results clearly indicate that miR-135b$5 \mathrm{p}$ inhibits LPS-induced TNF $\alpha$ production through activating AMPK.

\section{U937 cells with miR-135b-5p}
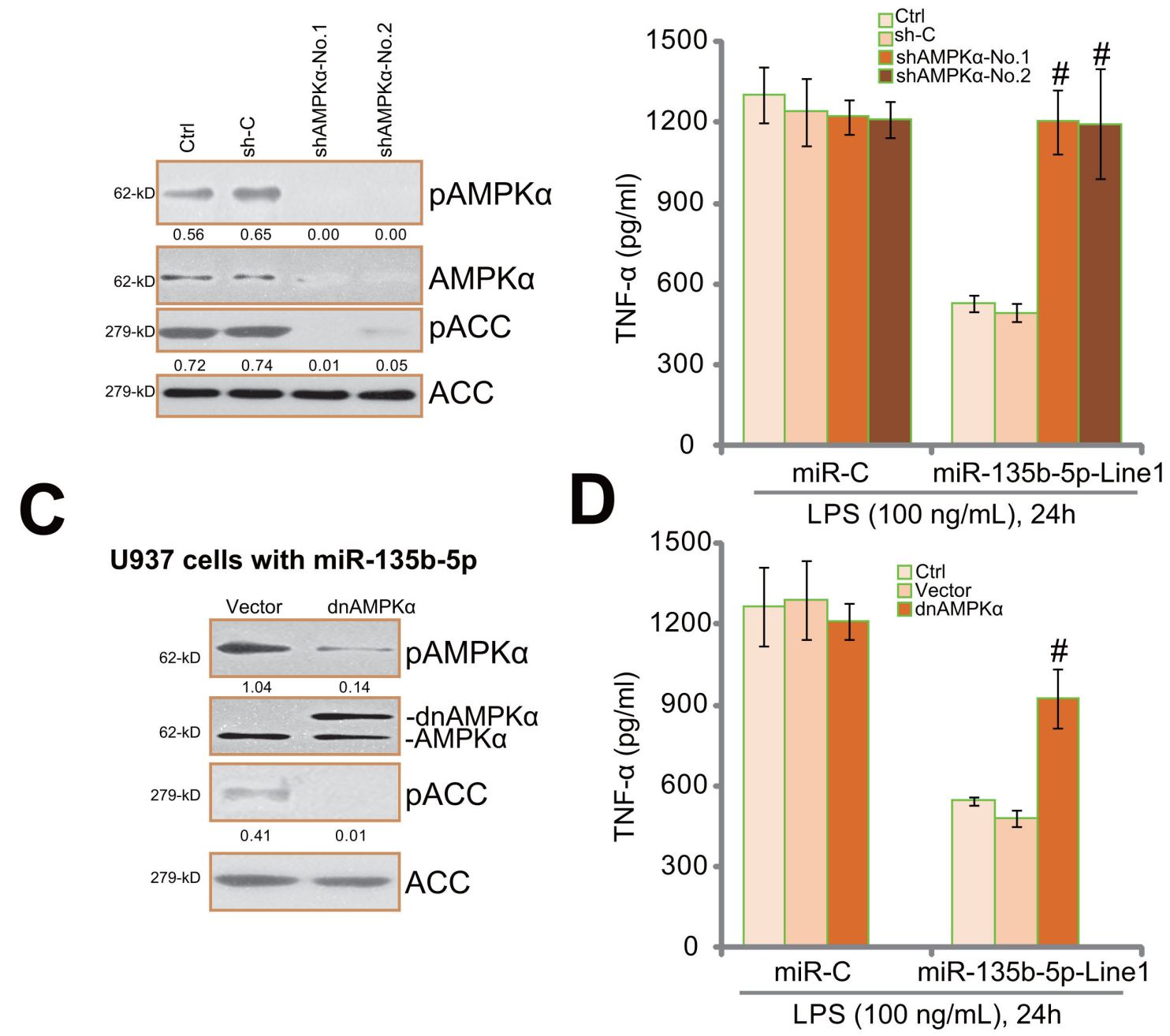

Figure 4: AMPK activation is required for miR-135b-5p's inhibition on LPS-induced TNF $\alpha$ production. miR-135b-5p expressing U937 cells were constructed with AMPK $\alpha$ shRNA ("shAMPK $\alpha$-No.1"/“shAMPK $\alpha$-No.2") or scramble control shRNA ("shC"), and stably cells were established; Expressions of listed proteins in these cells were tested by Western blot assay A.; Cells were treated with LPS (100 ng/mL) for 24 hours, TNF $\alpha$ production was tested B. miR-135b-5p expressing U937 cells were constructed with dominant negative AMPK $\alpha$ (T172A, "dnAMPK $\alpha$ ”, GFP-tagged) or empty vector ("pSuper-puro"), stably cells were established; Expressions of listed proteins in these cells were tested by Western blot assay C.; LPS-induced TNF $\alpha$ production was also examined D. Experiments in this figure were repeated for three times, and similar results were obtained. AMPK $\alpha$ and ACC phosphorylations were quantified (vs. regular ACC, A and C). "Ctrl" stands for un-transfected control cells (B and D). " $p<0.05$ vs. "sh-C" (B). " $p<0.05$ vs. "Vector" (D). 
miR-135b-5p inhibits LPS-induced ROS production, NFKB activation and TNF $\alpha$ mRNA expression

Existing evidences have shown that LPS induces production of reactive oxygen species (ROS), which is required for subsequent $\mathrm{NF} \kappa \mathrm{B}$ activation and TNF $\alpha$ mRNA expression [17, 27]. Interestingly, AMPK activation may function as an anti-oxidant signaling within a number of stress conditions [17, 26, 28-30]. We therefore analyzed ROS level and NFkB signaling in human macrophages with/our miR-135b-5p expression. As shown in Figure 5A, treatment of LPS in U937 cells induced significant ROS production, which was largely attenuated with miR-135b-5p expression (Figure 5A). Intriguingly, miR-135b-5p-induced anti-oxidant function also relies on AMPK activation (Figure 5A). AMPKa shRNA knockdown or mutation almost abolished miR135b-5p's ROS scavenging activity (Figure 5A).

Intriguingly, LPS-induced NFKB activation, tested by Western blot assay of $\mathrm{pIKK} \alpha / \beta(\operatorname{Ser} 176 / 180)$ (Figure 5B) and p65 DNA-binding assay (Figure 5C) [9], was significantly inhibited with miR-135b-5p expression. Consequently, LPS-induced TNF $\alpha$ mRNA expression was also attenuated (Figure 5D). Such effects by miR-135b$5 p$ were again almost abolished with AMPK $\alpha$ knockdown or mutation (Figure 5B-5D). Collectively, these results indicate that miR-135b-5p activates AMPK to inhibit LPS-induced ROS production, NFאB activation and TNF $\alpha$ mRNA expression.

\section{DISCUSSION}

LPS is sensed by CD14 and LPS-binding protein (LBP), and binds to its receptor Toll-like receptor 4 (TLR4 ) on monocytes [31,32]. This will lead to the recruitment of several key adaptor proteins (MyD88, TRAF6 and others) to activate downstream NFKB signaling cascade $[31,32]$. ROS production also plays a pivotal role in the process. Sanlioglu et al., showed Rac1-dependent ROS production induced LPS-induced NFKB activation and TNF $\alpha$ production [27]. On the other hand, ROS scavengers could attenuate LPS-induced inflammatory response [27]. For instance, Shen et al., demonstrated that cordycepin inhibited LPS-induced ROS production and subsequent TNF $\alpha$ production [17]. In the present study, we showed that miR-135b-5p activated AMPK signaling to inhibit LPS-induced ROS production and subsequent $\mathrm{NF} \kappa \mathrm{B}$ activation. This could be one key reason of TNF $\alpha$ inhibition by miR-135b-5p.

Existing evidences have implied AMPK as an antioxidative signaling under a number of stress conditions $[17,29,30]$. AMPK activation by energy depletion could attenuate oxidative stress via increasing NADPH content [30]. In this regard, AMPK-ACC signaling activation inhibits ROS accumulation via increasing NADPH production [30]. A recent study by She et al., demonstrated that AMPK activation could decrease $\mathrm{H}_{2} \mathrm{O}_{2}$-induced oxidative damages [29]. Recently, Zhang's group showed that cordycepin suppressed LPS-induced ROS production and NFKB activation through activating AMPK-NADPH signaling [17]. Our recent unpublished work showed that GSK621, a novel AMPK activator [16], attenuated LPSinduced ROS production, $\mathrm{NF \kappa B}$ activation and subsequent $\mathrm{TNF} \alpha$ expression (Wu et al., unpublished studies). In line with these findings, we show that AMPK activation by miR-135b-5p decreased LPS-induced ROS production and NFKB activation. Such effects by miR-135b-5p were almost abolished with AMPK inhibition. Thus, we propose that miR-135b-5p activates AMPK to attenuate LPS-induced ROS production, and subsequent $\mathrm{NF \kappa B}$ activation, which then inhibit TNF $\alpha$ mRNA expression and production.
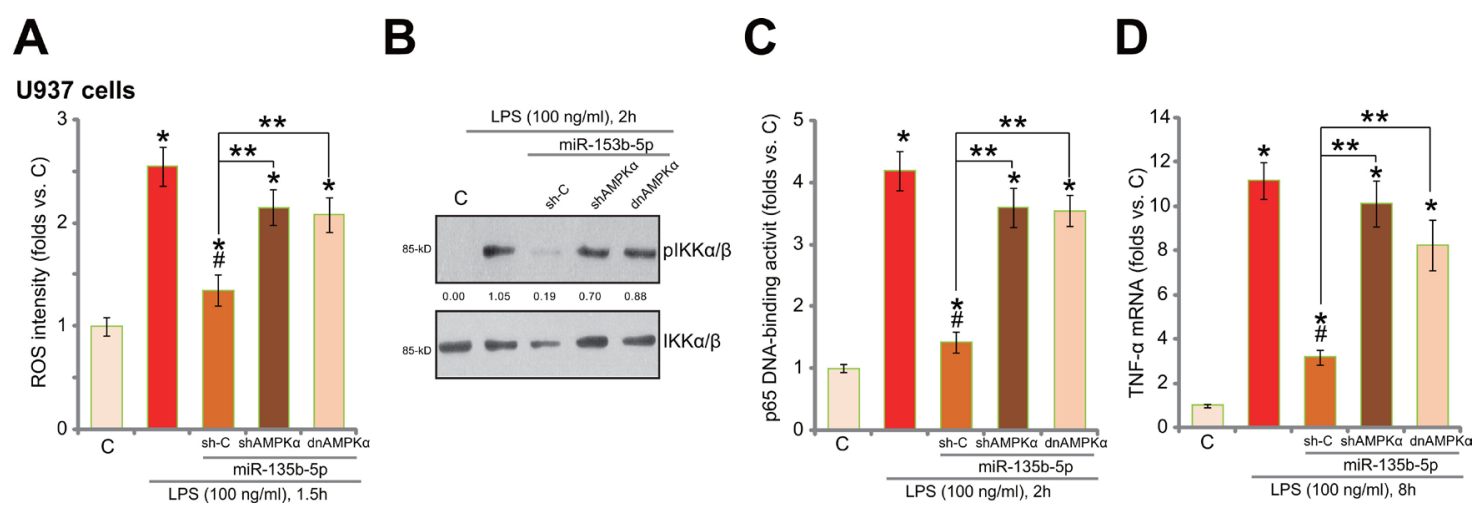

Figure 5: miR-135b-5p inhibits LPS-induced ROS production, NFkB activation and TNF $\alpha$ mRNA expression. miR135b-5p expressing U937 cells were constructed with scramble control shRNA ("sh-C"), AMPK $\alpha$ shRNA ("shAMPK $\alpha$ ", No.1) or dominant negative AMPK $\alpha$ (T172A, "dnAMPKa"), these cells or the control U937 cells were treated with LPS (100 ng/mL) or medium control ("C") for applied time, relative ROS intensity A., NFKB activation B and C. and TNF $\alpha$ mRNA expression D. were tested by listed assays. Experiments in this figure were repeated for three times, and similar results were obtained. IKK $\alpha / \beta$ phosphorylation was quantified (B). ${ }^{*} p<0.05$ vs. "C" group (A, C and D). ${ }^{*} p<0.05 v s$. LPS only group (A, C and D). ** $p<0.05$ (A, C and D). 
Although many AMPK activators have been developed thus far [33], there are few of them are being tested in clinical stages for various disease. The results of this study showing AMPK activation by miR-135b-5p via downregulating Ppmle provide a new strategy to activate AMPK and to inhibit LPS inflammatory responses.

\section{MATERIALS AND METHODS}

\section{Chemicals and antibodies}

LPS, puromycin and neomycin were purchased from Sigma Chemicals (Shanghai, China). All the antibodies utilized in this study were purchased from Cell Signaling Technology (Danvers, MA). The cell culture reagents were purchased from Hyclone of Thermo Fisher Scientific (Shanghai, China).

\section{Cell culture}

The human monocyte cell lines, U937 and THP-1, were purchased from the Cell Bank of Fudan University (Shanghai, China). Cells were cultured in RPMI 1640 supplemented with $10 \% \mathrm{FBS}$ and $1 \% \mathrm{~L}$-glutamine at $37^{\circ} \mathrm{C}$.

\section{Real-Time PCR assay}

The protocol of real-time reverse transcriptase quantitative polymerase chain reaction (RT-qPCR) was described in detail in our previous study [9]. The comparative $\mathrm{Ct}$ method $\left(2^{-\Delta \Delta \mathrm{Ct}}\right)$ was applied to calculate relative mRNA expression level [34]. Glyceraldehyde3-phosphate dehydrogenase (GAPDH) was tested as the reference gene. The primers for TNF $\alpha$ (F: 5'-GGAGGGGTCTTCCAGCTGGAGA-3', and R: 5'-CAATGATCCCAAAGTAGACCTGC-3') and GAPDH (F: 5'-AGGCTAGCTGGCCCGATTTC-3', and R: 5'-TGG CAACAATATCCACTTTACCAGA-3') were utilized. The primers for Ppm1e were described in other studies [35]. The expression of mature hsa-miR-135b-5p was evaluated by the TaqMan microRNA assay as described [36]. Five ng of total RNA was reverse-transcribed using TaqMan MicroRNA Reverse Transcription Kit (Applied Biosystem, Shanghai, China) [36]. All the primers and sequences were synthesized by Genepharm (Shanghai, China).

\section{Forced miR-135b-5p expression}

The pSuper-neo expression vector withmiR-135b-5p (based on sequence [36]) was provided by Dr. Cui's group at Nantong University (Nantong China) [25]. U937 or THP-1 cells were transfected with miR-135b-5p construct $(0.10 \mu \mathrm{g} / \mathrm{mL}$ of each well) through the Lipofectamine 2000 protocol (Invitrogen, Shanghai, China) for 24 hours. Stably cells were then selected by neomycin $(0.25 \mu \mathrm{g} /$ $\mathrm{mL}$ ) for two weeks. miR-135b-5p expression in the stable cells was tested by the RT-qPCR assay. Control cells were transfected with non-sense scramble microRNA-control ("miR-C") (Also provided by Dr. Cui [25]).

\section{TNF $\alpha$ enzyme-linked immunosorbent assay (ELISA) assay}

Following treatment of cells, TNF $\alpha$ content in the conditional medium was evaluated via the TNF $\alpha$ ELISA kit (R\&D Systems, Abingdon, UK) as described [9].

\section{Western blots}

As described [9], the protein lysates (20 $\mu \mathrm{g}$ per sample) were separated by SDS-PAGE gel, and were transferred onto PVDF membranes, which were then probed with primary and secondary antibodies. Enhanced chemiluminescence (ECL, Amersham, Shanghai, China) regents were utilized to detect targeted bands. The total gray of each protein band was quantified by Bio-Rad Quantity One software, and was normalized to corresponding loadings [9].

\section{shRNA knockdown}

The two lentiviral AMPK $\alpha$ short hairpin RNAs (shRNAs, "No1" and "No2", with non-overlapping sequences) were provided by Dr. Lu's group at Nanjing Medical University [37-39]. The two different Ppm1e lentiviral shRNAs (“-1/-2”) were provided by Dr. Cui's group at Nantong University. The lentiviral shRNA was added to cultured cells for 24 hours, and stably cells were selected by puromycin $(1.0 \mu \mathrm{g} / \mathrm{mL})$ for $10-14$ days [37-39]. Knockdown of AMPK $\alpha$ or Ppm1e was verified by Western blot assay. Control cells were infected with non-sense control shRNA lentiviral particles (Santa Cruz Biotech).

\section{AMPK $\alpha$ dominant negative mutation}

The pSuper-puro construct with dominant negative (T172A) AMPK $\alpha$ and the empty vector were provided by Dr. Lu's group at Nanjing Medical University [37, 40]. Lipofectamine 2000 was applied to transfect mutant AMPK $\alpha$ or the vector to miR-135b-expressing U937 cells. Stable cells were again selected by puromycin $(1.0 \mu \mathrm{g} /$ $\mathrm{mL}$ ). AMPK $\alpha$ mutation was verified by Western blot assay.

\section{Reactive Oxygen Species (ROS) assay}

ROS production was measured by dichlorofluorescin (DCF) oxidation assay as described [17]. Briefly, after applied treatment, cells were incubated with $10 \mu \mathrm{M}$ of DCFH-DA (Invitrogen) for $30 \mathrm{~min}$. Cells were then washed, trypsinized and resuspended in PBS. DCF fluorescence intensity was then tested using a FACS BD machine. The fluorescent intensity value of treatment group was expressed as fold changes of the control group. 


\section{Measuring NFKB (p65) DNA-binding activity}

The detailed protocol of this assay was described in our previous study [9]. Briefly, after treatment of cells, NFkB (p65) DNA-binding activity, analyzing from $1.0 \mu \mathrm{g}$ of cell nuclear extracts, was examined using the TransAM ${ }^{\mathrm{TM}}$ ELISA kit (Active Motif, Carlsbad, CA) with the manufacturer's protocol. The OD value of treatment group was always normalized to that of control group.

\section{Statistics analysis}

The statistical analyses were performed via the SPSS software (18.0), with $p<0.05$ taken as significant. Data were expressed as mean \pm standard deviation (SD). For comparisons among multiple groups, two-way ANOVA with the Bonferroni post hoc testing was performed.

\section{ACKNOWLEDGMENTS}

This work is partly supported by the NSFC.

\section{CONFLICTS OF INTEREST}

The authors declare no conflicts of interest.

\section{Author contributions}

Ping Li and Jian-bo Fan contributed equally to this study. All authors carried out the experiments, participated in the design of the study and performed the statistical analysis, conceived of the study, and participated in its design and coordination and helped to draft the manuscript. All authors read and approved the final manuscript.

\section{REFERENCES}

1. Barnes PJ. New anti-inflammatory targets for chronic obstructive pulmonary disease. Nat Rev Drug Discov. 2013; 12:543-559.

2. Brusasco V, Martinez F. Chronic obstructive pulmonary disease. Compr Physiol. 2014; 4:1-31.

3. Roversi S, Roversi P, Spadafora G, Rossi R, Fabbri LM. Coronary artery disease concomitant with chronic obstructive pulmonary disease. Eur J Clin Invest. 2014; 44:93-102.

4. Cosio MG, Saetta M, Agusti A. Immunologic aspects of chronic obstructive pulmonary disease. N Engl J Med. 2009; 360:2445-2454.

5. Lamela J, Vega F. Immunologic aspects of chronic obstructive pulmonary disease. N Engl J Med. 2009; 361:1024.

6. Rabinovich RA, Figueras M, Ardite E, Carbo N, Troosters T, Filella X, Barbera JA, Fernandez-Checa JC, Argiles JM,
Roca J. Increased tumour necrosis factor-alpha plasma levels during moderate-intensity exercise in COPD patients. Eur Respir J. 2003; 21:789-794.

7. Profita M, Chiappara G, Mirabella F, Di Giorgi R, Chimenti L, Costanzo G, Riccobono L, Bellia V, Bousquet J, Vignola AM. Effect of cilomilast (Ariflo) on TNF-alpha, IL-8, and GM-CSF release by airway cells of patients with COPD. Thorax. 2003; 58:573-579.

8. Ouagued M, Martin-Chouly CA, Brinchault G, LeportierComoy C, Depince A, Bertrand C, Lagente V, Belleguic C, Pruniaux MP. The novel phosphodiesterase 4 inhibitor, CI-1044, inhibits LPS-induced TNF-alpha production in whole blood from COPD patients. Pulm Pharmacol Ther. 2005; 18:49-54.

9. Li P, Wu Y, Li M, Qiu X, Bai X, Zhao X. AS-703026 Inhibits LPS-Induced TNFalpha Production through MEK/ ERK Dependent and Independent Mechanisms. PLoS One. 2015; 10:e0137107.

10. Hardie DG, Ross FA, Hawley SA. AMPK: a nutrient and energy sensor that maintains energy homeostasis. Nat Rev Mol Cell Biol. 2012; 13:251-262.

11. Tang J, Feng Y, Tsao S, Wang N, Curtain R, Wang Y. Berberine and Coptidis rhizoma as novel antineoplastic agents: a review of traditional use and biomedical investigations. J Ethnopharmacol. 2009; 126:5-17.

12. Shen J, Liang L, Wang C. Perifosine inhibits lipopolysaccharide (LPS)-induced tumor necrosis factor (TNF)-alpha production via regulation multiple signaling pathways: new implication for Kawasaki disease (KD) treatment. Biochem Biophys Res Commun. 2013; 437:250-255.

13. Zhao X, Zmijewski JW, Lorne E, Liu G, Park YJ, Tsuruta Y, Abraham E. Activation of AMPK attenuates neutrophil proinflammatory activity and decreases the severity of acute lung injury. Am J Physiol Lung Cell Mol Physiol. 2008; 295:L497-504.

14. Cacicedo JM, Yagihashi N, Keaney JF, Jr., Ruderman NB, Ido Y. AMPK inhibits fatty acid-induced increases in NF-kappaB transactivation in cultured human umbilical vein endothelial cells. Biochem Biophys Res Commun. 2004; 324:1204-1209.

15. Ducommun S, Ford RJ, Bultot L, Deak M, Bertrand L, Kemp BE, Steinberg GR, Sakamoto K. Enhanced activation of cellular AMPK by dual-small molecule treatment: AICAR and A769662. Am J Physiol Endocrinol Metab. 2014; 306:E688-696.

16. Sujobert P, Poulain L, Paubelle E, Zylbersztejn F, Grenier A, Lambert M, Townsend EC, Brusq JM, Nicodeme E, Decrooqc J, Nepstad I, Green AS, Mondesir J, et al. Co-activation of AMPK and mTORC1 Induces Cytotoxicity in Acute Myeloid Leukemia. Cell Rep. 2015; 11:1446-1457.

17. Zhang JL, Xu Y, Shen J. Cordycepin inhibits lipopolysaccharide (LPS)-induced tumor necrosis factor (TNF)-alpha production via activating amp-activated 
protein kinase (AMPK) signaling. Int J Mol Sci. 2014; 15:12119-12134.

18. Mihaylova MM, Shaw RJ. The AMPK signalling pathway coordinates cell growth, autophagy and metabolism. Nat Cell Biol. 2011; 13:1016-1023.

19. Shaw RJ, Kosmatka M, Bardeesy N, Hurley RL, Witters LA, DePinho RA, Cantley LC. The tumor suppressor LKB1 kinase directly activates AMP-activated kinase and regulates apoptosis in response to energy stress. Proc Natl Acad Sci U S A. 2004; 101:3329-3335.

20. Hardie DG. AMP-activated/SNF1 protein kinases: conserved guardians of cellular energy. Nat Rev Mol Cell Biol. 2007; 8:774-785.

21. Jensen TE, Rose AJ, Jorgensen SB, Brandt N, Schjerling P, Wojtaszewski JF, Richter EA. Possible CaMKK-dependent regulation of AMPK phosphorylation and glucose uptake at the onset of mild tetanic skeletal muscle contraction. Am J Physiol Endocrinol Metab. 2007; 292:E1308-1317.

22. Herrero-Martin G, Hoyer-Hansen M, Garcia-Garcia C, Fumarola C, Farkas T, Lopez-Rivas A, Jaattela M. TAK1 activates AMPK-dependent cytoprotective autophagy in TRAIL-treated epithelial cells. EMBO J. 2009; 28:677-685.

23. Voss M, Paterson J, Kelsall IR, Martin-Granados C, Hastie CJ, Peggie MW, Cohen PT. Ppm1E is an in cellulo AMPactivated protein kinase phosphatase. Cell Signal. 2011; 23:114-124.

24. Ambros V. The functions of animal microRNAs. Nature. 2004; 431:350-355.

25. Fan JB, Ruan JW, Liu W, Zhu LQ, Zhu XH, Yi H, Cui SY, Zhao JN, Cui ZM. miR-135b expression downregulates Ppmle to activate AMPK signaling and protect osteoblastic cells from dexamethasone. Oncotarget. 2016; 7:70613-70622. doi: 10.18632/oncotarget.12138.

26. Guo S, Mao L, Ji F, Wang S, Xie Y, Fei H, Wang XD. Activating AMP-activated protein kinase by an alpha1 selective activator Compound 13 attenuates dexamethasoneinduced osteoblast cell death. Biochem Biophys Res Commun. 2016.

27. Sanlioglu S, Williams CM, Samavati L, Butler NS, Wang G, McCray PB, Jr., Ritchie TC, Hunninghake GW, Zandi E, Engelhardt JF. Lipopolysaccharide induces Rac1-dependent reactive oxygen species formation and coordinates tumor necrosis factor-alpha secretion through IKK regulation of NF-kappa B. J Biol Chem. 2001; 276:30188-30198.

28. Zheng K, Lu H, Sheng Z, Li Y, Xu B. Low-concentration of perifosine surprisingly protects cardiomyocytes from oxygen glucose deprivation. Biochem Biophys Res Commun. 2016; 469:753-760.
29. She C, Zhu LQ, Zhen YF, Wang XD, Dong QR. Activation of AMPK protects against hydrogen peroxide-induced osteoblast apoptosis through autophagy induction and NADPH maintenance: New implications for osteonecrosis treatment? Cell Signal. 2014; 26:1-8.

30. Jeon SM, Chandel NS, Hay N. AMPK regulates NADPH homeostasis to promote tumour cell survival during energy stress. Nature. 2012; 485:661-665.

31. Bryant CE, Spring DR, Gangloff M, Gay NJ. The molecular basis of the host response to lipopolysaccharide. Nat Rev Microbiol. 2010; 8:8-14.

32. Miller SI, Ernst RK, Bader MW. LPS, TLR4 and infectious disease diversity. Nat Rev Microbiol. 2005; 3:36-46.

33. Hardie DG, Ross FA, Hawley SA. AMP-Activated Protein Kinase: A Target for Drugs both Ancient and Modern. Chem Biol. 2012; 19:1222-1236.

34. Livak KJ, Schmittgen TD. Analysis of relative gene expression data using real-time quantitative PCR and the 2(-Delta Delta C(T)) Method. Methods. 2001; 25:402-408.

35. Hentges KE, Kyttala M, Justice MJ, Peltonen L. Comparative physical maps of the human and mouse Meckel syndrome critical regions. Mamm Genome. 2004; 15:252-264.

36. Xu Z, Han Y, Liu J, Jiang F, Hu H, Wang Y, Liu Q, Gong Y, Li X. MiR-135b-5p and MiR-499a-3p Promote Cell Proliferation and Migration in Atherosclerosis by Directly Targeting MEF2C. Sci Rep. 2015; 5:12276.

37. Lu PH, Chen MB, Ji C, Li WT, Wei MX, Wu MH. Aqueous Oldenlandia diffusa extracts inhibits colorectal cancer cells via activating AMP-activated protein kinase signalings. Oncotarget. 2016; 7:45889-45900. doi: 10.18632/ oncotarget.9969.

38. Chen MB, Jiang Q, Liu YY, Zhang Y, He BS, Wei MX, Lu JW, Ji Y, Lu PH. C6 ceramide dramatically increases vincristine sensitivity both in vivo and in vitro, involving AMP-activated protein kinase-p53 signaling. Carcinogenesis. 2015; 36:1061-1070.

39. Lv G, Zhu H, Zhou F, Lin Z, Lin G, Li C. AMP-activated protein kinase activation protects gastric epithelial cells from Helicobacter pylori-induced apoptosis. Biochem Biophys Res Commun. 2014; 453:13-18.

40. Chen MB, Zhang Y, Wei MX, Shen W, Wu XY, Yao C, Lu $\mathrm{PH}$. Activation of AMP-activated protein kinase (AMPK) mediates plumbagin-induced apoptosis and growth inhibition in cultured human colon cancer cells. Cell Signal. 2013; 25:1993-2002. 